\title{
Acquisition and evaluation of radiometrically comparable multi-footprint airborne LiDAR data for forest remote sensing
}

\section{Korpela, Ilkka}

2017-06-01

Korpela , I 2017 , ' Acquisition and evaluation of radiometrically comparable multi-footprint airborne LiDAR data for forest remote sensing ' , Remote Sensing of Environment, vol. 194 , pp. 414-423 . https://doi.org/10.1016/j.rse.2016.10.052

http://hdl.handle.net/10138/308416

https://doi.org/10.1016/j.rse.2016.10.052

cc_by_nc_nd

acceptedVersion

Downloaded from Helda, University of Helsinki institutional repository.

This is an electronic reprint of the original article.

This reprint may differ from the original in pagination and typographic detail.

Please cite the original version. 


\section{Abstract}

\section{Introduction}

Forest inventories comprise observations, models and sampling. Airborne LiDAR has established its role in providing observations of canopy geometry and topography. These data are input for estimation of important forest properties to support forestry-related decision-making. The primary deficiency in forest remote sensing is tree species identification. This study examines the quite atypical option of using multi-footprint airborne LiDAR data. Features of such sensor design exist in recently introduced multispectral laser scanners. The first objective was to acquire radiometrically normalized, multi-footprint (11, 22, 44 and $59 \mathrm{~cm}$ ) waveform (WF) data that characterize 1064-nm backscatter reflectance on the interval scale. The second objective was to analyze and validate the data quality in order to draw the correct conclusions about the effect of footprint size. Finally, the data were analyzed in different forest canopies. The experiment was carried out in Finland. Footprint variation was generated by acquiring data at different flying heights and by adjusting the transmitted power. The LiDAR campaign was successful and the data were of sufficient quality, except for a $1 \mathrm{~dB}$ trend due to the atmosphere. Significant findings were made concerning the magnitude of atmospheric losses, the linearity of the amplitude scale and the bandwidth characteristics of the receiver, the stability of the transmitter, the precision of the amplitude data, the transmission losses in canopies and power lines as well as the response of WF attributes to footprint size in forest canopies. Multi-footprint data is a promising approach although the species-specific signatures were weak.

Keywords: Canopy structure, Tree species, Forest inventory, Laser scanning, Waveform, Normalization, Linearity, Impulse response, Losses

Airborne LiDAR data are used for many purposes, and the rationales for the present study originate from LiDAR remote sensing (RS) of forests (Vauhkonen et al., 2014). It has recently become an integral part of forest management planning systems in Finland (Maltamo and Packalén, 2014). LiDAR is an observation tool that has reduced the sampling intensity and provided entirely new observations for the estimation of forest attributes. In Finland, forest planning inventories consist of area-based estimation of stand boundaries, attributes and management proposals, using a combination of field reference, aerial image and LiDAR data, all finally followed by a field inspection. The data acquisition costs have decreased compared with field-work intensive systems, with both improvements and deficiencies in the deliverables. The primary deficiency in forest RS, not only in 
Finland, is tree species identification. There is a need for observations that aid in solving this problem. Multi-footprint data may

31 comprise one option.

32 Regarding sensors, foresters have mostly used data acquired by topographic small-footprint instruments. Narrow beams 33 promote accurate geometry and reach the ground. While simulation studies have highlighted how sensor properties influence 34 the available information (Disney et al., 2010, Hovi \& Korpela, 2014), certain constraints pertain to sensor design. They include 35 the need for extremely high dynamic range, consideration of eye-safety, receiver sensitivity and bandwidth, availability of powerful lasers at different wavelengths, data transfer and storage capacity requirements, power consumption, limitations on the size of the mirror and the aperture, and the final instrument cost. Advances in the last 10-15 years relate to improvements in pulse repetition frequency, capture of waveform (WF) data, fast direct georeferencing and the detection of multiple echoes, inter alia. Teledyne Optech (Vaughan, Ontario) recently released a three-band, dual-footprint WF-recording sensor called Titan, in which the divergence of the 532-nm pulses is wider than that of 1064-nm and 1550-nm pulses. The relative reflectance difference of green and woody vegetation between the 1064 and $1550 \mathrm{~nm}$ bands is likely beneficial for tree species identification as the green/woody silhouette visible from the above shows between-species differences owing to differences in leaf angle distributions, and in branch and crown morphology. On the other hand, WFs carry species-specific traits (Hovi et al., 2015), when tens of pulses per crown are evaluated. How the combination of WF and multi-band data will benefit species identification, is a very interesting topic. Although advances are foreseeable with multispectral LiDAR, this study examines the relatively unconventional concept of multi-footprint LiDAR to investigate how such data characterize canopy structure, and in general augment our understanding of the characteristics of LiDAR data. The rest of this section provides further background to the topic and introduces hypotheses that were tested empirically.

The radar equation (Eq. 1) applies to LiDAR as well, and states that the received power $\left(\mathrm{P}_{\mathrm{r}}\right)$ is influenced by the aperture area (D), the atmospheric transmission losses $(T)$, the optical efficiency of the system $(Q)$, the transmitted power $\left(P_{t}\right)$, the laser beam divergence $(\beta)$, the range $(\mathrm{R})$, and the backscattering cross-section of the target $(\sigma)$ :

$$
P_{r}=\frac{P_{t} D Q}{4 \pi \beta^{2}} T^{2} \frac{\sigma}{R^{4}}
$$

53 where $\sigma$ can be said to be comprised of target reflectance, geometry and the illuminated/silhouette area. Eq. (1) is 54 'instantaneous', while in reality the power terms and $\sigma$ are time- or distance dependent and the optical power of the beam has 55 a Gaussian cross-section profile (cf. Wagner et al., 2006; M allet and Bretar, 2009).

Eq. (1) states that $\mathrm{P}_{\mathrm{r}}$ is in linear dependence with target reflectance and the transmitted power. A large aperture increases $\mathrm{Pr}_{\mathrm{r}}$ and the signal-to-noise ratio (SNR), because the extra solar illumination reaching the detector, non-attenuated, is minor compared to the internal receiver noise. Atmospheric attenuation is $0.1-0.3 \mathrm{~dB} / \mathrm{km}$ in weather conditions that are suitable for LiDAR RS. 
The effect of R, i.e. the spherical losses, depend on the target, because large surfaces, linear targets and blob-like objects all

60

61 exhibit different response. A sensor that provides ratio- or interval-scale measurements of $P_{r}$, will show a fourfold (6 dB) increase in well-defined surfaces, when Ris halved, whereas $\operatorname{Pr}_{r}$ is 8 or 16 times ( 9 or $12 \mathrm{~dB}$ ) larger for a wire or a small leaf. Tree crowns are volumetric and the structure influences the signal change due to R (Korpela et al., 2010, Gatziolis, 2011), making range normalization ill-posed in canopies. In linear targets, such as cables, the distribution of $\operatorname{Pr}$ has an exponential-even shape with a modest peak near the maximum, while for blob-like features, the distribution is inverse-exponential.

Divergence defines the angular spread of the beam's Gaussian irradiance profile. Here it is defined by the $1 / \mathrm{e}^{2}$ point. A target may give rise to a measurable echo, only if it is illuminated by the pulse center. The backscatter cross-section may shrink for a tilted target, or at least the returning pulse is extended and dampened. Because sensors record meaningful data only, backscattering $\left(P_{r}\right)$ needs to be strong enough to trigger an observation, and the real signal distributions are always truncated by some combination of $\sigma$ and the receiver noise. The sensor used in this study stores a single continuous and fixed-length WF, the recording of which is triggered by the first threshold-exceeding echo. Some sensors record variable-length WF samples with pauses in between and thresholding applies to this 'piecewise WF-recording' as well (e.g. Armston et al., 2010). In general, weak backscattering preceding the first echo may remain undetected, while such weak within-canopy or ground scattering may be observable, when the recording is on, and missed, while it is paused. This constitutes a vertical sampling bias. A somewhat similar effect causes selection bias in the analyses of discrete-return (DR) intensity data, when the separation between only and first-of-many echoes is often made (Ørka et al., 2010; Korpela et al., 2010b), because this instrument-driven division is influenced by the backscatter cross-section profile of targets following the first interaction, for example the ground flora in the case of tree crowns.

Regarding multi-footprint data, changing solely the beam width will not influence $\mathrm{P}_{\mathrm{r}}$ as long as the target is a well-defined surface filling the footprint, while for small targets, $\operatorname{Pr}$ varies according to the intersection geometry, as stated. Multiple scattering and transmission losses are introduced instantly following the first pulse-canopy interaction (the subsequent targets comprise an apparent differential backscatter cross-section profile (Richter et al., 2015), high-order volumetric scattering can be significant, and backscattering occurs far from the beam path (Hovi and Korpela, 2014). Foliage orientation, size, density and silhouette area were shown to influence the recorded intensity in broadleaved and coniferous trees in Finland (Korpela et al., 2010b, 2013; Hovi and Korpela, 2014). If beam width increases by a factor of $i$, while $P_{t}$ is constant, the average $P_{r}$ decreases by $1 / i^{2}$ and $1 / i$ in blob-like and linear targets, respectively. A wide beam is more likely to find directional canopy gaps and the proportion of pulses that illuminate the ground is higher. Many small targets that trigger an echo in narrow beams remain undetected in large-footprint data and contribute to transmission losses, which explain why ground signal levels can be expected to be lower in large-footprint data. The wider the pulse, the smaller is the likelihood for the pulse to reach the ground with maximal energy. Thus, measuring the reflectance properties of the forest floor using single-return data is more accurate in 
narrow footprint data as shown for understory lichens in Korpela (2008). The peak amplitude distribution of canopy echoes can

91 therefore be expected to be shifted towards low values in large footprint data. It can also be expected that the return WFs are 92 widened in large-footprint data, because a wider beam illuminates targets that also spread across a larger depth.

93 As stated, spherical losses depend on the target geometry and it is therefore evident that a fixed signal-to-noise-ratio (SNR) 94 cannot be maintained for the canopy and the ground, when scanning from different heights (Goodwin et al., 2006, Ørka et al., 95 2010). When R is doubled, a $6 \mathrm{~dB}$ increase in $P_{t}$ will maintain the SNR for well-defined surfaces. We cannot expect this to hold true for the forest floor, because canopy transmission losses will increase with increasing footprint size. The SNR of small targets will fall even if the power is increased by $6 \mathrm{~dB}$, which explains the reduction in canopy echo counts in high-altitude LiDAR

98 (Goodwin et al., 2006). Of course, atmospheric losses introduce additional signal loss. Varying the scan zenith angle changes $\mathrm{R}$ and the incidence angles. Pulses reflecting from tilted surfaces will exhibit widened echoes, in which the peak amplitude is dampened, and both the widening and the dampening depend on the footprint size (e.g. M allet and Bretar, 2009).

Increasing $\mathrm{P}_{\mathrm{t}}$ improves the SNR. Other options include enlarging the receiver aperture and mirror, or decreasing the receiver noise level. The latter comprises dark noise of the detector, electronic and digitization noise as well as natural light that reaches the detector. The energy captured by an aperture that is located in the footprint center, and focuses light to the retina, cannot exceed critical values, which in turn limits $P_{t}$ and/or beam divergence. Eye-safety is a more severe concern at $532 \mathrm{~nm}$ than at $1064 \mathrm{~nm}$ or $1550 \mathrm{~nm}$. Regarding multi-band sensors, we can note that the reflectance of foliage at $532 \mathrm{~nm}$ is one tenth of that at $1064 \mathrm{~nm}$. Given the same aperture, divergence and output power, the green band signals will thus be 10 times lower. In order to improve the SNR of well-defined targets (at $532 \mathrm{~nm}$ ), it is possible to increase both the transmitted power and/or beam divergence, where the latter may be needed for eye-safety. Because multiple scattering in vegetation is weak in visible bands, the returning $532 \mathrm{~nm}$ pulses are probably less extended compared to NIR pulses of the same beam divergence, which may be exploited in target classification using multi-band LiDAR.

Radiometric observables in LIDAR data include intensity and WF amplitude data. The instantaneous spectral irradiance is captured by the aperture, reflected to a collimating lens that directs it to the photon detector through a band-pass filter. The detector and the circuits that follow, i.e. the receiver, have certain noise characteristics, impulse response and bandwidth (BW) properties. The latter two determine how the dynamics of the photon surge are captured. Receiver bandwidth is inherently limited and rapid changes in the photon surge are low-pass filtered in the WF. As long as the transmitted pulses are stable, minor deficits in bandwidth are not critical for reliable ranging, which is also shown in this study. Receiver performance may depend on signal strength and for example very strong echoes can temporarily increase the noise level or cause 'ringing', i.e. 'ghost echoes', in the WF (e.g. Armston et al., 2010). If the internal signal levels are too high, i.e., outside the linear (dynamic) range of the receiver circuits, the sensor of this study performs erroneous discrete ranging and the intensity data are distorted. 
A one-nanosecond sampling interval is typical in WF-recording sensors, and the length (full width at half maximum, FWHM) of the transmitted pulse is below 10 ns. The power goes up to several kWs in some nanoseconds only, but the exact power envelope of the transmitted pulse is usually not known. Any within- or between-pulse variation directly influences the convolution of the transmitted pulse with the backscatter cross-section profile of the targets. Some sensors provide a digitized sample of the transmitted pulse to enable exact ranging and echo attribute retrieval in post processing (Wagner et al., 2006; Roncat et al., 2014)

Radiometrically quantitative analyses require that the properties of the transmitted pulse (directional target illumination) and the receiver's response to instantaneous at-sensor irradiance (measurement of reflected radiance) are known, in addition to the properties of the medium. Such absolute calibration is typically unattainable for LiDAR sensors and vicarious calibration with reflectance targets becomes the only option (cf. Wagner, 2010). This in turn is challenging because reference measurements of zero phase-angle reflectance are hard to establish and are rarely done. A linear response of the receiver is usually assumed (with gain and offset), or alternatively a look-up-table is used. Some sensors have two receivers and the model is needed for both. DR sensors can have individual circuits for the detection of echoes and the intensity values can have different calibration. The sensor used in this study has a single receiver, in which the gain of the signal chain is dynamically adjusted up to $3 \mathrm{~dB}$ by an automatic gain control (AGC) circuit. In order to draw conclusions about the influence of footprint size, using the WF data, it is vital to establish a mapping between the amplitude data and the at-sensor power. Such mapping was performed in this study.

This study examines the relatively unconventional concept of multi-footprint LiDAR to investigate how such data characterize canopy structure, and in general augment our understanding of LiDAR data. Specific objectives were:

1. Plan and carry out WF LiDAR data acquisition with a fixed beam divergence sensor that results in radiometrically comparable multi-footprint data in well-defined targets.

2. Carry out radiometric normalization of peak amplitude data using well-defined surfaces to remove the effects of the AGC circuit, to estimate the offset of the amplitude scale and to identify any deficits in the acquisition.

3. Validate the normalization across the study site to ensure that the data qualifies for analyses on the influence of the footprint size.

4. Acquire independent data using the same sensor to investigate and verify whether the amplitude scale of the receiver is in linear dependence with the instantaneous at-sensor power.

5. Investigate the impulse response of the receiver to assure that WF attributes are influenced only by the pulse-target interaction.

6. Carry out empirical tests to show that the hypotheses presented in the introduction are confirmed by the measured real data. 


\section{$151 \quad 2.1$ Research site}

The experiments were carried out in Hyytiälä $\left(61^{\circ} 50^{\prime} \mathrm{N}, 24^{\circ} 17^{\prime} \mathrm{E}\right)$, southern Finland. The area is mostly covered by forest, but there are also fields, open and forested peatlands, built environment and a network of forest roads. Aerial photographs cover the time period of 1946-2015 and there are 19 airborne LiDAR datasets acquired in 2004-2015. Geometric reference data include close-range photogrammetric images, surveying points and profiles. These data as well as the redundant airborne image and LiDAR data were used for geometric quality control of the LiDAR data sets used here. (Hovi, 2015).

\subsection{Waveform-recording LiDAR data}

Objective \#1 was to acquire multi-footprint data, in which the same energy was transmitted by varying the beam divergence. Sensors with the option of changing divergence were not available. To produce the same effect, a Leica ALS60 sensor was used at different heights as its transmitted power can be adjusted by almost $20 \mathrm{~dB}(1-100 \%)$. The nadir $1 / \mathrm{e}^{2}$ footprint diameters were $11,22,44$ and $59 \mathrm{~cm}$ from the acquisition heights of $0.5,1.0,2.0$ and $2.7 \mathrm{~km}$, respectively. The pulse power was set to nominal levels of 100, 55, 14 and 4\% (Tables 1 and 2) to account for the spherical losses in well-defined surfaces. Strip overlaps were increased for the higher altitudes to reach comparable pulse density. According to the manufacturer, the FWHM of the transmitted pulses is 4 or 9 nanoseconds, which showed as 7 and $10 \mathrm{~ns}$ in the recorded data (Fig. 1). The wavelength is $1064 \mathrm{~nm}$, and Fig. 2 shows the difference of intensity data in different targets between the ALS60 and a Riegl LM S-Q680i sensor, which operates at $1550 \mathrm{~nm}$. The Riegl data was acquired in 2013 (not tabulated) and was used for illustrations only. The same ALS60 sensor was used for datasets 2012_\#\# and 2013_\#\# (Table 2). The acquisition in 2013 was carried out to verify the linear response of the receiver, objective \#4. It comprised three flying heights: $700 \mathrm{~m}, 800 \mathrm{~m}$ and $900 \mathrm{~m}$. The sensor was operated in an unorthodox manner by deliberately fixing both the transmitter power and the receiver gain (AGC, see later). Appropriate gain and power settings were found by first acquiring the 700 -m data with the AGC on. These datasets have $\pm 12.5 \%$ range variation.

171 The receiver front-end in the ALS60 implements an AGC and the gain can vary by $0-3.2 \mathrm{~dB}$ (typically less) in response to scene brightness so that the internal signal levels are kept in the linear region of the range detection circuits. As said, the gain can also be fixed (2013_\#\# datasets). The influence of the AGC can largely be removed, using an 8-bit 'voltage value' that is stored for each pulse, shown first in Korpela (2008). 
Table 1. Sensor parameters.

\begin{tabular}{lr}
\hline Wavelength, nm & 1064 \\
Pulse length, FWHM , ns & 4,9 \\
System WF, FWHM, ns & $7.8,10.2$ \\
WF of transmitted pulse & No \\
Divergence, mrad, 1/e 2 & 0.22 \\
M irror & Oscillating \\
Front-end amplifier & Automatic Gain Control \\
\hline Triggering of WF-recording & Discrete-return circuit \\
WF-recording sequence & Single, 256 samples \\
WF Sampling rate, ns & 1 or 2 \\
LiDAR position, XYZ & yes \\
Discrete-return echoes & on-the-fly; 1, 2, 3 or 4 \\
Echo attributes & range, XYZ, intensity \\
Acquisition height, m & $500,1000,2000,2700$ \\
\hline
\end{tabular}

Table 2. Characteristics of the experimental LiDAR acquisitions. See also Table 1.

\begin{tabular}{lll}
\hline Dataset & $2012 \_(05,10,20,27)$ & $2013 b_{-}(07,08,09)$ \\
\hline Purpose & Multi-footprint data & Linearity tests \\
Date, local & July 5,2012 & June 15-16, 2013 \\
Time, GMT & $19-22$ & $21-00$ \\
Phenology & mid-season & early season \\
Sensor & ALS60 & ALS60 \\
Altitude, m & $500,1000,2000,2700$ & $700,800,900$ \\
WF density per m² & $5,5,3,2$ & $6,6,5$ \\
Scan angle, degrees & \pm 15 & \pm 15 \\
PRF, kHz & $152,99,59,45$ & 106 \\
System WF, FWHM, ns & $7.8,10.1,10.3,10.4$ & 7.8 \\
Footprint, 1/e ${ }^{2}, m$ & $0.11,0.22,0.45,0.59$ & $0.15,0.18,0.20$ \\
AGC & on & off \\
\hline
\end{tabular}

ALS60 is primarily a DR sensor and the WF storage calls for an optional digitizing module. The storage is triggered by the first DR echo. A sequence of 256 amplitude (A) values is recorded including a buffer of 30 samples (Fig. 1). Two oscilloscopes take turns in digitizing the pulses at $1 \mathrm{GS} / \mathrm{s}$. Their bandwidth (BW) is $300 \mathrm{M} \mathrm{Hz}$ and the rise time for a square signal from $10 \%$ to $90 \%$ is 1.2 

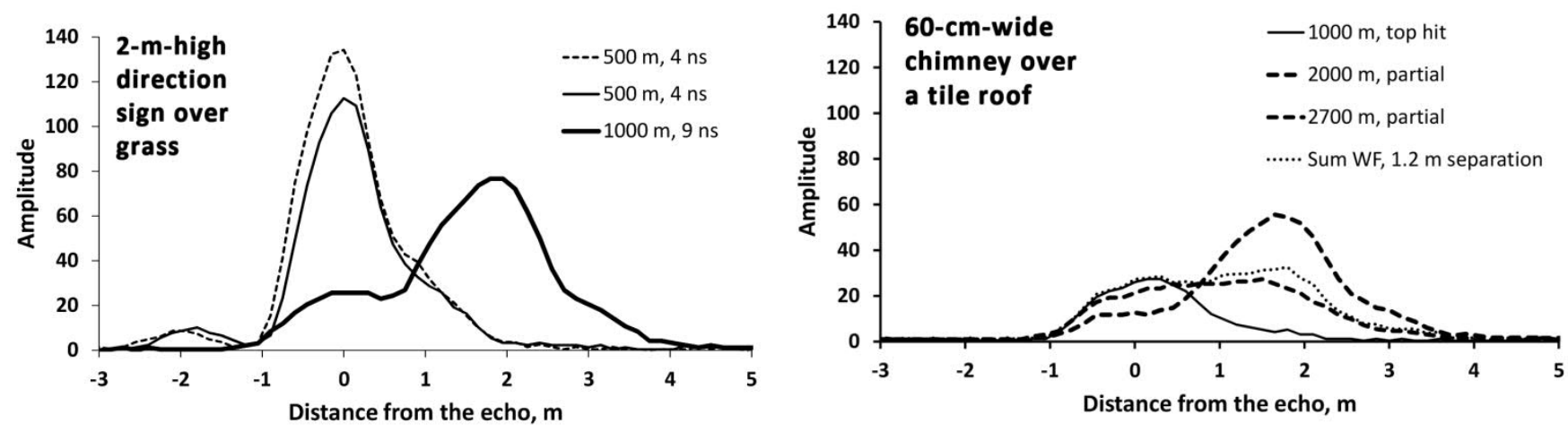

Fig. 1. The x-axis shows the range in meters centered $(0 \mathrm{~m})$ at the first echo. In the left figure there are three single-echo WFs.

The 500-m echoes are from the ground, while the 1000-m echo was triggered $2 \mathrm{~m}$ above the ground despite the strong ground signal. Weak backscattering from the signs is seen even for the $500-\mathrm{m}$ pulses in the preceding buffer. In the figure on the right there are three pulses that gave rise to an echo from the top of the chimney. The 1000-m pulse reflected 'entirely' from the top, while the 2/2.7 km pulses have scattered from the chimney and the roof. The 'Sum WF' is the 1000-m WF summed with its

195 shifted $(6 \mathrm{~ns}, 90 \mathrm{~cm})$ copy.

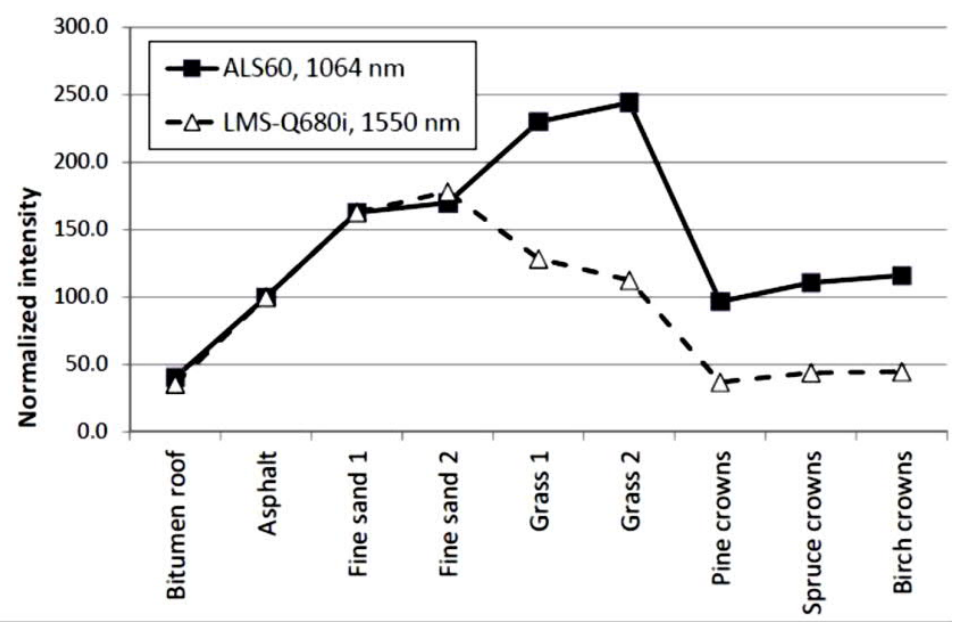

Fig. 2. Averaged and normalized peak amplitude values (asphalt=100) in 1064 and $1550 \mathrm{~nm}$ pulses (not tabulated, Riegl LM SQ680i sensor, 700 m AGL, amplitude data linearized using a look-up-table) in different surfaces. Major reflectance differences of green vegetation are seen between 1064 and $1550 \mathrm{~nm}$. Backscattering from tree crowns is not only influenced by the reflectance properties but also by footprint size and the foliage orientation and crown structure. Footprint diameters here are comparable, $0.44 \mathrm{~m}$ and $0.40 \mathrm{~m}$. 
The WF attributes were computed in a straightforward way for what were called noise-exceeding amplitude sequences (NEAS, also referred to as an echo). M ore advanced methods for detecting individual echoes that may contribute to a NEAS, and for the derivation of echo attributes such as amplitude and width are discussed in e.g. Roncat et al. (2014). A detailed description of the calculations is given in Hovi et al. (2015). In Hovi et al. (2015) these were found important in tree species identification.

Noise was defined as the variation around a baseline signal, during which the receiver recorded background illumination only. The AGC was a particular challenge in defining the baseline, as the gain influenced both the baseline and the noise. The baseline varied by up to 0.3 amplitude units, which is only $0.25 \%$ of the maximum amplitude and neglecting it did not influence the results. The threshold for the start and end of a NEAS was three standard deviations of the observed variation around the baseline (Fig. 3).

In brief, the WF is first moderately low-pass filtered. The NEAS is found by tracing the (interpolated) points where the signal arises from and falls into the noise. The highest amplitude in a NEAS is the peak amplitude, pA, which is in strong dependence with discrete-return intensity, but only in well-defined targets. The sum of the amplitude values is energy, E. The width attributes were FWHM and L FWHM was computed for the highest peak (defining the half maximum) and Lis the total length of a NEAS. The rise speed, riS, was assumed to describe 'the porosity' of the illuminated target, and was defined as the ratio of the rise time and that of a pulse reflecting from a well-defined surface having the same $\mathrm{pA}$. The normalization is needed as the above-the-noise rise time depends on the signal strength (c.f. constant fraction discriminator in range detection).

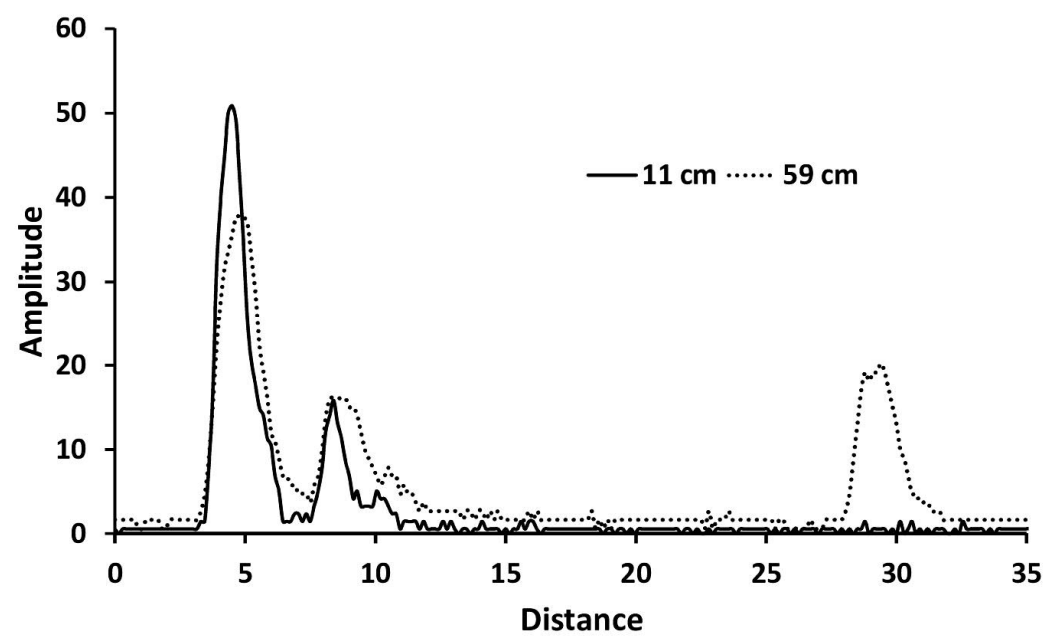

Fig. 3. WFs of two adjacent and collinear pulses in a pine forest. The 59-cm WF is shifted up by one unit to be visible. The first echoes have reflected from a branch $24.7 \mathrm{~m}$ high and had an XYZ separation of $0.16 \mathrm{~m}$, while the ground intersection points had an XY separation of $0.17 \mathrm{~m}$. The scan zenith angle was 8 degrees. Ground echo can be seen for the wider pulse. The attribute values for the first NEASs are 50 and 37 for $\mathrm{pA}, 8.3$ and 12.6 for FWHM, 1.16 and 1.16 for RiS, and 486 and 518 for $\mathrm{E}$. 


\subsection{Radiometric normalization of the multi-footprint data}

As stated, the AGC amplifier is a particular property of the receiver in the ALS60. In the datasets used, the relative gain was 1-2.1, i.e. 0-3.2 dB in power, assuming that the intensity and amplitude values are in linear dependence with at-sensor power.

The influence of the AGC was the same on amplitude and intensity data. The $A G G_{\text {voltage }} \times$ gain relationship resembles a sigmoid, and the parameters of a normalization function ( $f_{A G G}$ a 2 nd-degree polynomial) were estimated using homogenous surfaces of varying reflectance (Fig. 4). These surfaces were bitumen roof, old asphalt, fine sand and grass. Fig. 4 shows the uncorrected data for grass.

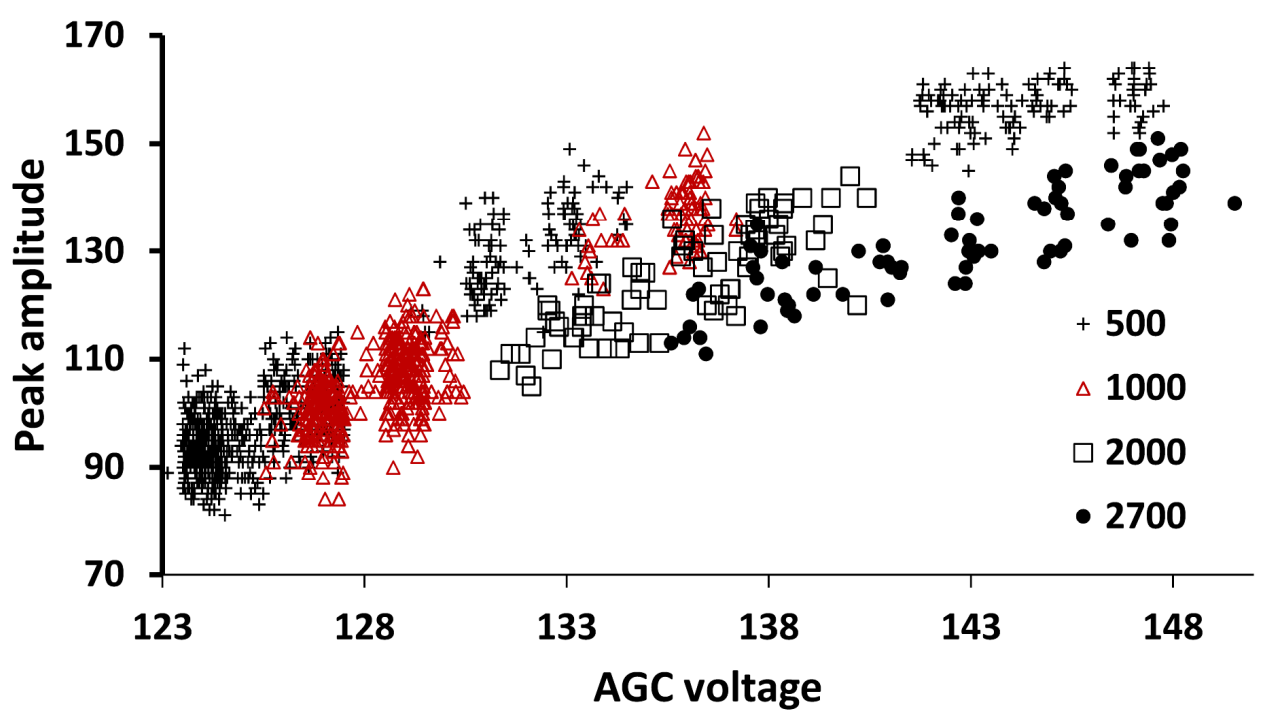

Fig. 4. Uncorrected peak amplitude $x$ AGCuoltage distributions of pulses reflecting from grass in the four 2012_\#\# datasets. The sigmoid shape is seen in the $500 \mathrm{~m}$ data.

The normalizing model (Eq. 2) of a pulse i, acquisition height $j$ and sensor $k$, used the explanatory variables R, AGGoltage and the scan zenith angle. The parameters to be solved were the offset of the amplitude, the parameters of $f_{A G c}$ as well as coefficients $d$ for each acquisition height that absorb possible deviations of the transmitted power, recalling that the power could only be set at $1 \%$ nominal intervals.

$$
A_{\text {norm }(i, j, k)}=\left(\frac{R_{i}}{R_{\text {ref }(k)}}\right)^{2} \cdot \frac{1}{f_{A G C(k)}} \cdot\left(A_{o b s(i)}-\text { offset }_{k}\right) \cdot \cos \left(\theta_{i}\right) \cdot d_{j}
$$

The estimation of $\mathrm{f}_{\mathrm{AGC}}$ was carried out by first minimizing a weighted sum of coefficients of variation and trend coefficients of univariate regression, indicating removal of trends, over all surfaces and datasets. At this phase $80-95 \%$ of the within-surface variance was removed. Parameters offset and $d_{j}$ were finally estimated by minimizing per surface mean amplitude differences of different acquisition heights in each surface (Fig. 5). In the solution, $d_{j}$ were 1.00, 3.56, 12.94, and 22.03 and the offset of the A 
scale was 11.4. If the power had been set perfectly and atmospheric attenuation was zero, the expected values for $d_{j}$ are 1,4 , 16, and 29.16. Unfortunately, it was realized at this phase that the flight planning software did not account for atmospheric losses. Using the $d_{j}$ values, attenuation was assessed to be $0.22 \pm 0.06 \mathrm{~dB} / \mathrm{km}$, which is realistic for $1064 \mathrm{~nm}$. The $0.06 \mathrm{~dB} / \mathrm{km}$ residual standard deviation shows that losses explain the deviations only partly. Power adjustment was done at $1 \%$ intervals, which corresponds to $25 \%$ relative accuracy in the $500 \mathrm{~m}$ data. Overall, the data showed an approximately $1 \mathrm{~dB}$ trend from 0.5 to $2.7 \mathrm{~km}$, which is also visible in Fig. 4 as 'vertical layers'.

Parameter estimation (vicarious calibration) of Eq. 2 could not be done by constraining with the absolute reflectance differences of the calibration surfaces, as only HCRF (hemispherical-conical reflectance factor) measurements at $900 \mathrm{~nm}$ by a field spectrometer were available. These measurements were shown to match the 2012 AGC-corrected data reasonably well in Korpela et al. (2013). However, the between-surface differences in Fig. 5 are in the instrument scale. Thus, the linearity of the A data, with respect to power entering the aperture, had to be tested (Section 2.5).

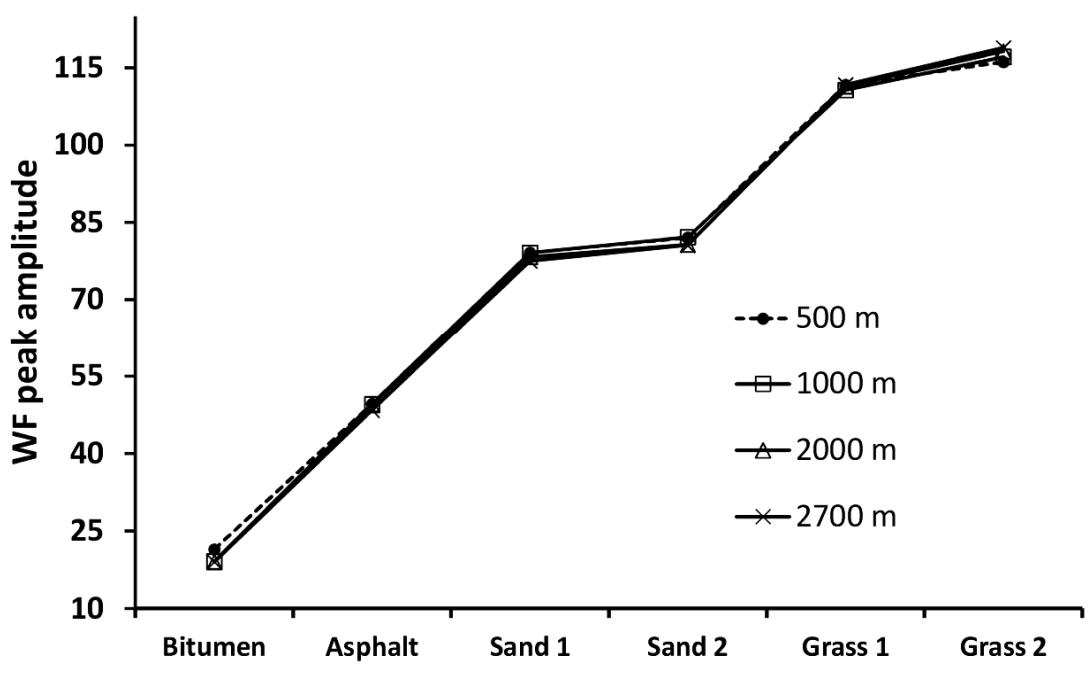

Fig. 5. Normalized mean (peak) amplitude values of the four acquisition heights in six calibration surfaces. The (dotted) 500-m data deviated in two cases more than $5 \%$, which could be due to strip-level variations of the low output power.

\subsection{Evaluation of the receiver's compliance with the radar equation}

Concerning the linearity of the ALS50/60 sensors, Korpela et al. (2010) compared the intensity data of Optech ALTM 3100 and Leica ALS50-ii sensors to assess the influence of vegetation structure in range correction, i.e., the value of pwr in the range normalization coefficient $\left(R / R_{\text {ref }}\right)^{p w r}$. The results with ALTM 3100 were in line with those by Gatziolis (2011) and the optimal pwr term was 2.1-2.8 for trees and could be logically linked to species-specific structure and echo type. Thus, the pwr term was close to 2 for 'only' echoes that are strong and was highest, nearly 2.8, for 'first-of-many' echoes in Scots pine that have the most 'diffuse' crowns. However, the ALS50-ii intensity data did not comply with the radar equation, and the optima were 
unclear with the pwr term even below 2. A simple linear model with zero offset was used, which for ALS50-ii (which is similar to ALS60) is now known to be incorrect (Fig. 6), while in ALTM 3100 the zero intensity was closer to the no-signal level.

The amplitude and intensity values in ALS60 were stored as 8-bit signed integers. 'No-signal sections' of the WFs, i.e., the front and tail parts of the 256-sample-long WFs, showed variance, which also responded to the AGC. The no-signal amplitude values were about 10-12, while the first-return intensity data was 'truncated' at zero (Fig. 6). The zero point (offset) of the first-return intensity was negative, and was estimated to be about -24 using Eq. (2), while the offset of A was 11.4 (Section 2.4). The estimation of the offset of the intensity scale was almost ill-posed, probably because the linear model (response to at-sensor power) is inaccurate, which is also indicated by Fig. 6.

The 2013_\# datasets were acquired to verify the linear response of the ALS60 receiver. In these data, the receiver gain and transmitted power were fixed. The pwr term of the R-normalization coefficient was again estimated using surfaces of varying reflectance. Fig. 7 implies that the peak amplitude data (restricted to pulses that had triggered a DR echo) are in a non-linear dependence with the power entering the aperture. Fig. 8 shows a power $\rightarrow$ A mapping that creates the pattern in Fig. 7. It deviates only slightly from a linear model. The component(s) that cause this modest non-linearity in the fixed receiver gain mode remain unidentified. Possibilities include the Avalanche photodiode and its transimpedance amplifier, other amplifiers and the digitizer. Furthermore, the non-linearity of low and high signals (Figs. 6, 7, 8) may be due to different components.

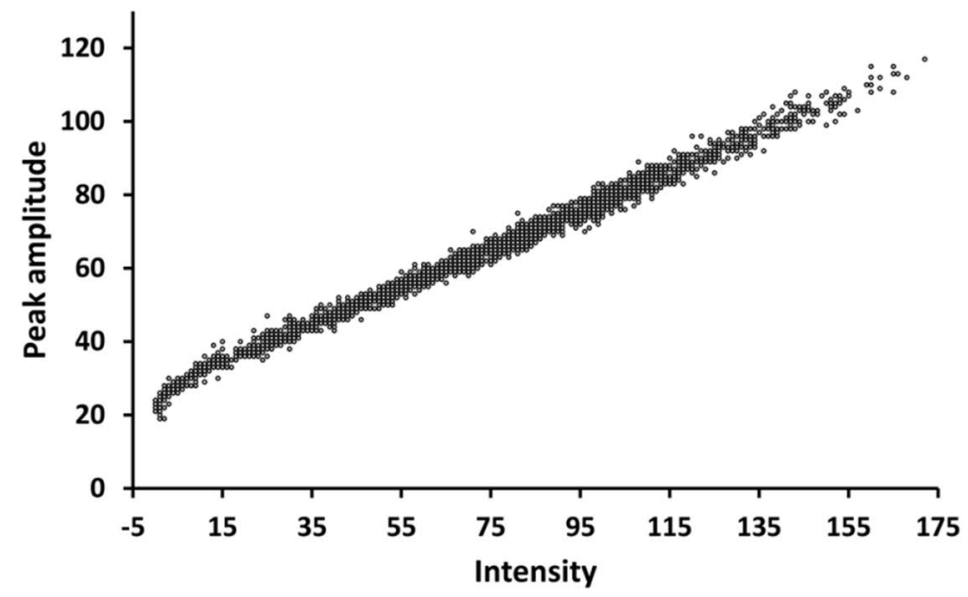

Fig. 6. The relationship between first-echo WF peak amplitude and DR intensity in a scene comprising bitumen, tile and metal roofs, short grass, concrete and asphalt. Echo triggering requires a pA of approximately 20. 


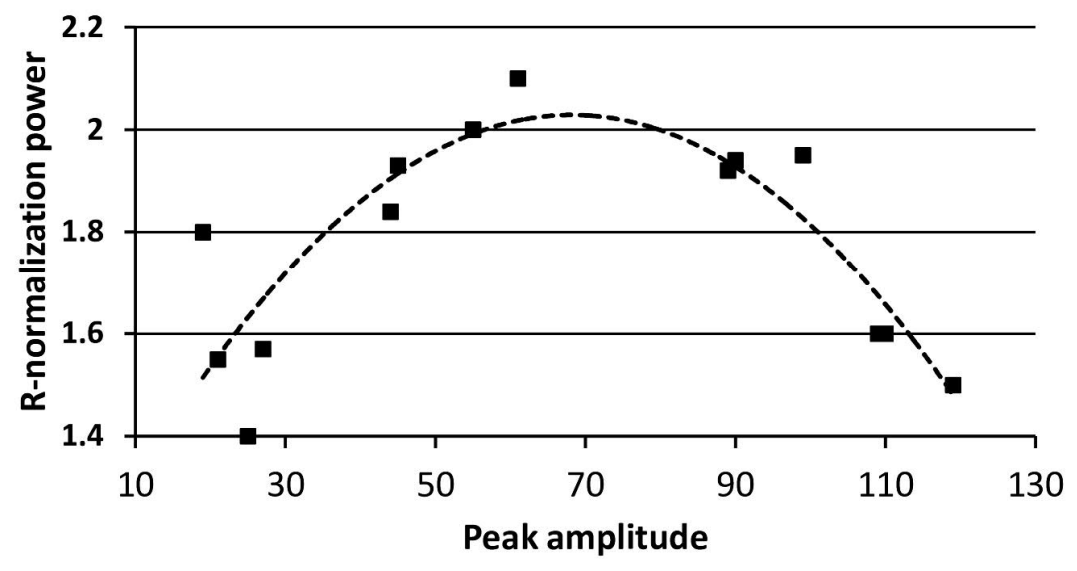

285 Fig. 7. Estimated power term of range normalization (y-axis) for the pAdata (x-axis) in the 2013_datasets. In a linear sensor the power factor is two. Analyses apply to horizontally aligned surfaces of varying reflectance in pulses that gave rise for an observation in $700 / 800,700 / 900$ and $800 / 900$ m pulses. The optimal power term minimized the squared sum of normalized differences for a particular surface and data pair. The observation is placed at the pA value corresponding to the $800 \mathrm{~m}$ acquisition.

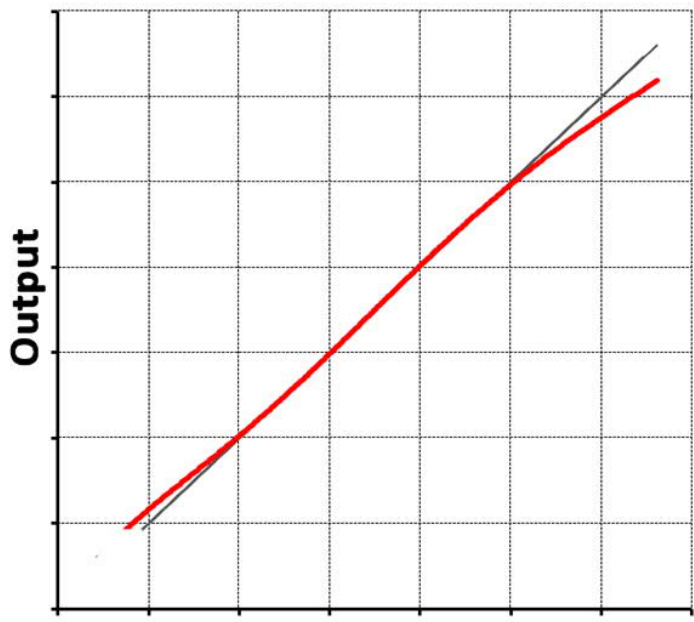

Input

Fig. 8. A non-linear sensor response curve that produces the pattern in Fig. 7. The $x$-axis depicts the power entering the aperture, while the y-axis shows the corresponding peak amplitude value.

\subsection{Variation of the received waveforms due to sensor effects}

The mean FWHMs in well-defined targets were $10.1-11.3 \mathrm{~ns}$ and $6.8-7.9 \mathrm{~ns}$ in the $22-59 \mathrm{~cm}$ and $11 \mathrm{~cm}$ footprint data, respectively. The dependence of FWHM on pA was different for the 4 and $9 \mathrm{~ns}$ pulses. FWHM increased moderately with pA in the 9 ns data, while it decreased considerably with increasing pA in the 4 ns data (Fig. 9). 

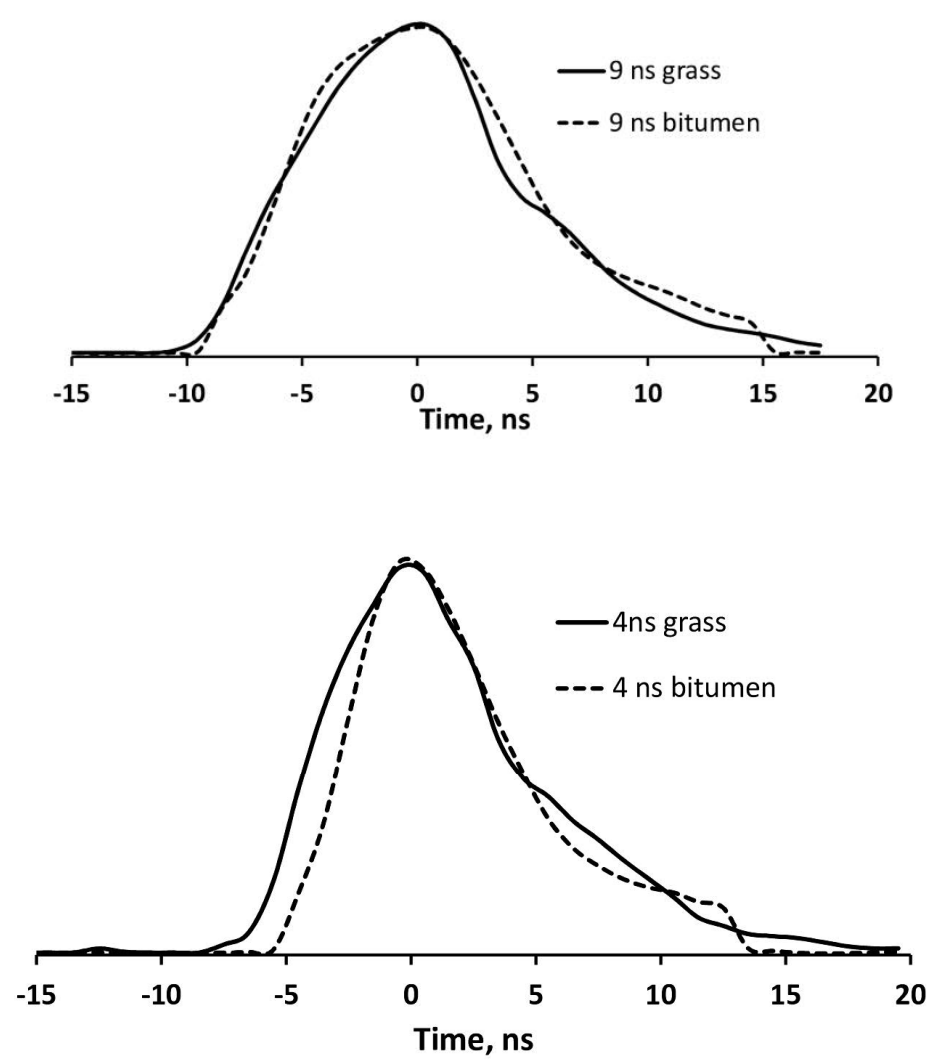

300 Fig. 9. Averaged, centered and normalized WFs of $4(7)$ and $9(10)$ ns pulses (transmitted, received). The response of FWHM to surface brightness differs with pulse length. In 4 ns pulses the recorded WF rises in 5-7 ns. The shape difference of the rising part of the 4 ns pulses is caused by the receiver's impulse response, which differs for weak and strong signals. The shape of the 9 ns pulses is retained better in bright and dark targets.

These findings imply that the receiver's response to the fast rising 4 ns pulses differed slightly between weak and strong signals.

The CV (coefficient of variation) of FWHM was 1-1.5\%. To compare, in some low (300 m) altitude datasets that were acquired in Hyytiälä with the Riegl LMS-Q680i sensor, the FWHM depends strongly on the signal strength, because of the saturation of the amplitude scale due to the low altitude, while $700 \mathrm{~m}$ data with the same sensor showed practically no FWHM $\times$ pA dependence. The echo width should depend only on the target properties, which was not exactly true in the ALS60 data used here.

\subsection{Evaluation of the radiometric normalization}

Radiometric normalization (Eq. 2) was validated across the landscape, using surfaces that were known from the field and/or identified in large-scale aerial images. Each surface was represented by more than 100 pulses per footprint size. The relative differences in Table 3 are normalized with respect to the 11-cm data. The differences were correlated between the 22, 44 and $59 \mathrm{~cm}$ data with $\mathrm{R}^{2}$ of $0.74,0.86$ and 0.89 , which implies that the $11 \mathrm{~cm}$ data did have offsets due to the within- or between-strip

315 fluctuation of the low (4\% of maximum) transmitted power. Using $9 \%$ of the maximum would have been at the eye-safety limit. Some of the 11-cm strips were repeated the following day with a 20\% (0.8 dB) higher pA. Table 3 also shows that the relative 
deviations were negatively correlated with the surface brightness, i.e., the pA values in dark surfaces and in the $22-59 \mathrm{~cm}$ data were modestly higher than in the $11 \mathrm{~cm}$ data. This can be caused by the different receiver response as the $11-\mathrm{cm}$ data had shorter pulses with a fast rise in the WF.

As stated, the 2012_\# acquisitions unintentionally did not account for atmospheric losses. The at-sensor threshold backscattering that triggers an echo depends on the instrument, not the acquisition height as such. The atmospheric losses however caused this threshold to be higher, in terms of the required target backscatter cross-section, in the high-altitude data. The effect was estimated to be $1.0 \mathrm{~dB}$ or $26 \%$ between the $500-\mathrm{m}$ and $2700-\mathrm{m}$ data (Section 2.4). Based on the $900-\mathrm{nm}$ field HCRF measurements of different bitumen surfaces that were carried out in 2008/2009 (Korpela et al. 2011), the minimal echotriggering 'reflectance' was about 0.03 in $11 \mathrm{~cm}$ data and 0.038 in $59 \mathrm{~cm}$ data. For this study I found an even darker bitumen roof, in which most of the $500 \mathrm{~m}$ pulses had produced an echo, while the probability reduced with acquisition height (** in Table This $0-1 \mathrm{~dB}$ 'echo-triggering bias' was thus present in all data. It could be observed in comparable (same LiDAR strips, adjacent targets) data that covered a dark and gently sloping bitumen roof and an adjoining meadow (** in Table 3 ). In comparison to the $11 \mathrm{~cm}$ data, the pAin the $22-59 \mathrm{~cm}$ data were $15 \%$ lower in bitumen, while the values for meadow were $1-4 \%$ higher. The roof had a reflectance variation due to litter (nearby trees), mosses and lichens (north vs. south exposure). The 11-cm data had more no-echo pulses, which means that the $11 \mathrm{~cm}$ samples were biased towards brighter roof patches, while the larger footprints averaged the variation. Overall, the validation in different surfaces showed that apart from very dark surfaces, the mean pA was not affected much by the footprint size (Table 4). Furthermore, the differences in the dark validation surfaces were in all likelihood caused by small scale reflectance variation. One of the dark surfaces was a pond with dense canopies of sub-emergent mosses next to a sedge fen. The same scale-related phenomenon was observed here. The mosses did not always give rise to an echo and the 11-cm targets probably comprise small, dense and drier moss patches. 
Table 3. Relative differences (\%) in peak amplitude in different validation surfaces. Values are normalized to the $11 \mathrm{~cm}$ data except for the first row. Values in which the peak amplitude was lower than in the $11 \mathrm{~cm}$ case are positive. Surfaces marked with * or $* *$ are adjacent and qualify for pair-wise comparisons. Surfaces \#1, \#2 and \#3 are presented in Section 2.10.

\begin{tabular}{lrrrrr}
\hline Surface & PA & $\mathbf{1 1}$ & $\mathbf{2 2}$ & $\mathbf{4 4}$ & $\mathbf{5 9}$ \\
\hline Field & 107 & - & 0.0 & -2.2 & 1.5 \\
& 115 & 0.0 & 2.1 & 1.6 & 3.8 \\
\hline Sedge fen & 98 & 0.0 & -4.2 & -6.7 & -5.2 \\
Low-sedge bog & 108 & 0.0 & 0.7 & -5.8 & -4.5 \\
Sedge fen * & 107 & 0.0 & -1.9 & -1.6 & -2.0 \\
Sub-emergent mosses* & 24 & 0.0 & 12.4 & 10.9 & 17.3 \\
\hline Fine sand & 87 & 0.0 & 0.5 & 1.4 & 2.1 \\
\hline Dark bitumen roof** & 17 & 0.0 & 12.9 & 15.2 & 15.1 \\
Meadow ** & 115 & 0.0 & -4.2 & -3.3 & -1.2 \\
\hline Forest roads & 66 & 0.0 & -2.0 & -2.4 & -1.9 \\
& 77 & 0.0 & 0.9 & 2.0 & 2.6 \\
\#1 & 80 & 0.0 & 0.5 & 3.0 & 2.9 \\
\#2 & 70 & 0.0 & 3.0 & 3.2 & 3.4 \\
& 73 & 0.0 & 1.1 & -4.4 & 2.2 \\
& 42 & 0.0 & 3.7 & 3.2 & 5.9 \\
\#3 & 50 & 0.0 & 3.2 & 2.5 & 4.3 \\
& 77 & 0.0 & -3.1 & -5.7 & -5.2 \\
\hline Asphalt roads & 51 & 0.0 & 4.8 & 4.2 & 6.4 \\
& 49 & 0.0 & 2.5 & 2.8 & 4.6 \\
& 42 & 0.0 & 0.5 & 0.4 & 2.9 \\
\hline
\end{tabular}

2.8 Scale-dependent reflectance variation

Table 4 shows the CV of pA in surfaces of varying small-scale variation. Asphalt and bitumen (different from that in Table 3) showed a stable CV. The between-pulse variation reduced with increasing footprint size in hay and in the mire surfaces owing to their decimeter-scale spatial variation. The mire was a mosaic of 20-60-cm wide dry hummocks surrounded by wetter white mosses and sedges. The multi-footprint data provided logical results. The lowest CV was $2.8 \%$. Bitumen showed relatively high CVs, because of the low relative measurement accuracy of weak signals. A comparison between the AGC on (and normalized) and AGC off acquisitions could be made with the 2013_07 dataset. The CVs were slightly lower in the AGC off data: $8.9 \rightarrow 8.6 \%$, 
normalization was effective and, conditioned on the stability of the targets, that the precision of the instrument was comparable between 2012 and 2013.

Table 4. Coefficient of variation (CV, \%) of peak amplitude in different man-made and natural surfaces. Surfaces are in decreasing order of brightness (About $0.45 \rightarrow 0.04$ in reflectance).

\begin{tabular}{llccc}
\hline \multicolumn{1}{c}{ Surface } & \multicolumn{5}{c}{ Footprint, $\mathbf{~ m ~}$} \\
& $\mathbf{1 1}$ & $\mathbf{2 2}$ & $\mathbf{4 4}$ & $\mathbf{5 9}$ \\
\hline Hay, <50 cm & 7.0 & 5.8 & 4.6 & 3.6 \\
Short grass & 4.9 & 4.3 & 4.0 & 3.5 \\
Mire surface & 9.7 & 8.3 & 6.3 & 5.4 \\
Fine sand & 4.5 & 3.5 & 3.0 & 2.8 \\
Asphalt, old & 7.4 & 7.3 & 6.9 & 7.2 \\
Asphalt, new & 5.1 & 4.9 & 5.0 & 5.1 \\
Gravel road & 11.4 & 9.7 & 7.7 & 6.9 \\
Bitumen & 9.4 & 9.4 & 9.0 & 8.6 \\
\hline
\end{tabular}

\subsection{Transmission losses in wires and tree crowns}

Next, the data was evaluated using careful measurements of transmission losses caused by three parallel (1.2 m separation, 152 $\mathrm{m}$ in total) power line cables $11 \mathrm{~m}$ above a spring barley field. The (bright) insulators in the poles were positioned using forward ray-intersection point estimates obtained in large-scale aerial images and the LiDAR points and constrained by the $1.2 \mathrm{~m}$ separation. Line-to-line distances were used to find pulses that had potentially intersected a cable. The limiting distance was the $95 \%$ point of the footprint diameter, i.e. $0.83,0.62,0.31$ and $0.16 \mathrm{~m}$ for the $59,44,22$ and $11 \mathrm{~cm}$ footprint diameters, respectively. Assuming that the pulse (intersection, 3D LiDAR positions were available) geometry was correct, that the irradiance profile of pulse cross-section is Gaussian, that the intersection probabilities were equal and that the cables had a $1.2 \mathrm{~m}$ separation, the theoretical losses for a cable of $1 \mathrm{~cm}$ thickness are $2.8,1.5,0.7$ and $0.8 \%$. The observed relative differences of $\mathrm{pA}$ were $2.6,1.6,0.7$ and $1.1 \%$ for the footprint sizes of $11,22,44$ and $59 \mathrm{~cm}$. The results were thus in line with expectations.

The transmission losses were next examined in an approximately 100-year-old barren pine stand having one dominant canopy layer only. Fig. 10 shows results of relative transmission losses measured by the peak amplitude of ground returns (of single return pulses) as a function of the $2 \mathrm{D}$ trunk-pulse distance at the $60 \%$ relative tree height, which approximates crown base height rather well in this stand. The crown width estimates $(\mathrm{N}=213)$ were obtained by fitting a surface of revolution to the LiDAR point cloud as in (Korpela et al., 2011; Hovi et al., 2015). The ground flora comprises three moss species and patches of reindeer lichens. Tree heights are 15.9-26.5 m. In Korpela (2008), the ground flora in this stand was mapped using a $960 \mathrm{~m}^{2}$ photo mosaic and no correlation was found in the spatial distribution of the bottom flora and the trees, i.e., the reflectance properties of the 
forest floor are spatially stationary. The pA of ground returns of pulses that had passed through the crown decreased with increasing footprint size (Fig. 10). Thus, less energy reached the ground when the footprint became larger and the single ground echo was weaker. Fig. 11 shows how the losses, as measured by energy of the first echo, correlate negatively with the peak amplitude of the ground return in two pine bogs. The other site has Sphagnum species and tussock cottongrass in the understory, while the ground flora of the other bog comprises a dense canopy of 30-60-cm-high wild rosemary. The reflectance differences of the low vegetation are reflected by the peak-amplitude values of the second echoes.

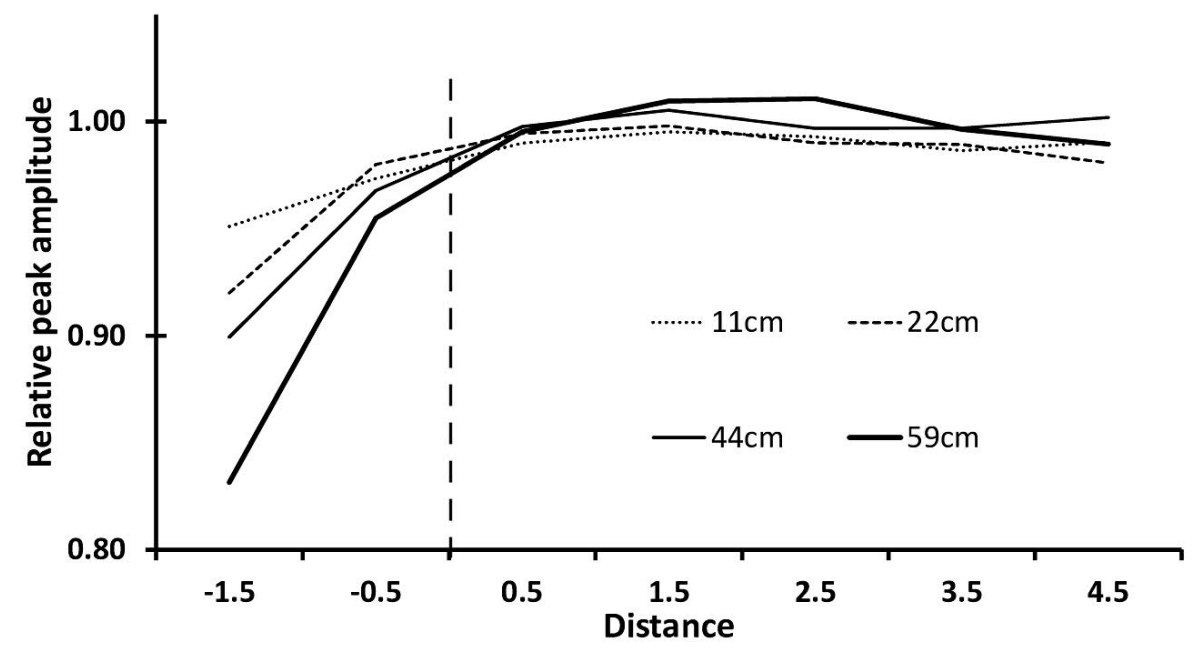

Fig. 10. Relative peak amplitude of (single) ground returns below a Scots pine canopy as a function of the pulse-trunk distance (m) at the crown base height. The amplitudes were normalized to data 3-5 $\mathrm{m}$ away from the trunks. A distance of zero corresponds to the case in which the pulse intersected the crown perimeter at the crown base height. A negative value means that the intersection was inside the crown model.

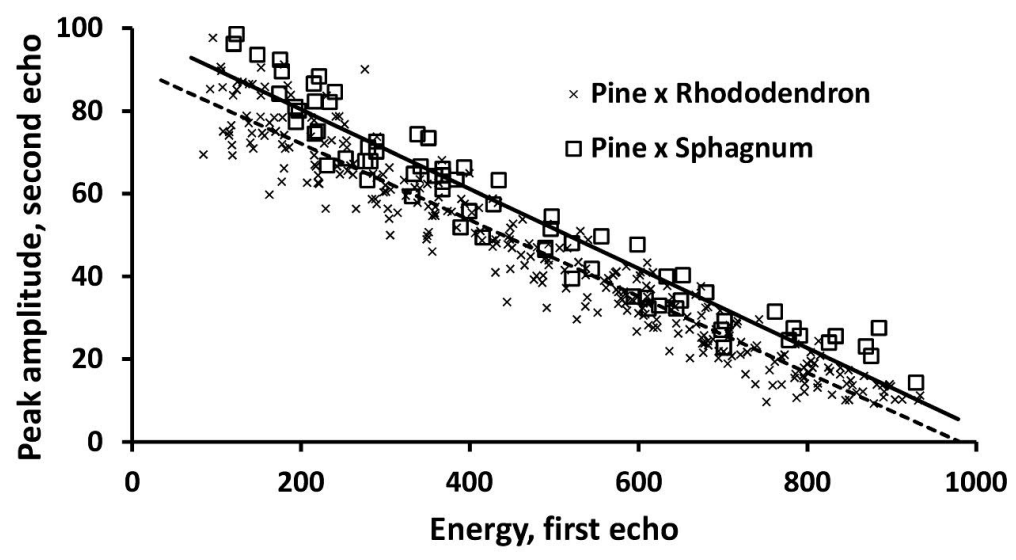

Fig. 11. Joint distributions of the energy of the first echo and the peak amplitude of the second echo in 9 ns pulses $(1,2$ and 2.7 $\mathrm{km}$ data) from two barren pine bogs that differ in understory flora. The pulses had a single WF peak in each of the two echoes. 


\subsection{WF features in single-species tree canopies}

Next, the response of WF attributes to the varying footprint diameter was evaluated in tree canopies. Three 30-year-old planted,

non-thinned, pure and dense stands of Scots pine, Norway spruce and Silver birch adjacent to a forest road were chosen. The road surface was essential for controlling between-dataset offsets in pA. The roads are marked as \#1, \#2 and \#3 in Table 3. The small relative offsets of $\mathrm{pA}$ were eliminated from the $\mathrm{pA}$ and $\mathrm{E}$ differences in Table 5. The energy $(\mathrm{E})$ attribute varied least with footprint size. The relative variance was also lowest in E with CVs ranging from 10 to 20\%. Interestingly, Eincreased by 8,6 and $7 \%$ with footprint size in pine, spruce and birch, respectively. The differences between species were largest in pA and $\mathrm{E}$, which is in line with the findings in Hovi et al. (2015). The relative decrease of pA with increasing footprint size is interesting, as it was lowest for birch (16.6\%), followed by pine (19.4\%) and spruce (25.9\%). An extra stand of Black alder showed a decrease of $12.6 \%$, but this stand did not have a control surface. These findings indicate weak species-specific 'signatures'.

Table 5. M ean values and CV (\%) of first-echo WF attributes in 30-year-old pure pine, spruce and birch stands. The LiDAR plots $\left(1300 \mathrm{~m}^{2}\right)$ contained approximately 70 trees. FWHMs were normalized to the mean values from well-defined surfaces. The E attributes of $11 \mathrm{~cm}$ (4 ns pulses) were multiplied by 1.307, which is the average ratio between $11 \mathrm{~cm}(4 \mathrm{~ns})$ and $9 \mathrm{~ns}$ data in welldefined surfaces of varying reflectance.

\begin{tabular}{lcllll}
\hline Species & Footprint & FWHM & RiS & pA & Energy \\
\hline \multirow{4}{*}{ Birch } & 11 & $1.51(38)$ & $1.35(28)$ & $66.1(34)$ & $1141(19)$ \\
& 22 & $1.49(38)$ & $1.30(25)$ & $67.9(30)$ & $1163(14)$ \\
& 44 & $1.68(40)$ & $1.51(26)$ & $58.7(29)$ & $1205(13)$ \\
& 59 & $1.77(39)$ & $1.63(25)$ & $55.2(28)$ & $1235(12)$ \\
\hline \multirow{5}{*}{ Spruce } & 11 & $1.33(40)$ & $1.21(26)$ & $60.2(33)$ & $941(20)$ \\
& 22 & $1.40(41)$ & $1.25(28)$ & $57.2(32)$ & $935(16)$ \\
& 44 & $1.66(47)$ & $1.45(30)$ & $49.6(34)$ & $982(15)$ \\
& 59 & $1.84(47)$ & $1.60(30)$ & $44.6(33)$ & $995(15)$ \\
\hline \multirow{4}{*}{ Pine } & 11 & $1.45(37)$ & $1.30(27)$ & $53.4(30)$ & $869(16)$ \\
& 22 & $1.49(35)$ & $1.32(24)$ & $51.8(25)$ & $875(12)$ \\
& 44 & $1.68(34)$ & $1.53(23)$ & $46.1(22)$ & $916(10)$ \\
& 59 & $1.77(37)$ & $1.63(23)$ & $43.1(25)$ & $932(13)$ \\
\hline
\end{tabular}

FWHM increased as footprint diameter increased, which is according to expectations. When the footprint size increased from 11 to $59 \mathrm{~cm}$ the relative increase in FWHM was $17 \%$ in birch, $38 \%$ in spruce and $22 \%$ in pine. The rise time attribute showed a similar pattern with smaller differences between species, compared to echo width, i.e. FWHM. Depending on the range detection algorithm, the increase of rise time can influence the height distribution of canopy echoes such that the wide footprint 
points have lower heights. This phenomenon was not tested here, because it would have required the implementation of a range detection algorithm and careful geometric calibration of the LiDAR data. It can also be seen that while pA decreases (dampens) with footprint size, Eincreases moderately as return pulses stretch.

\section{Discussion}

This study was a largely successful attempt to acquire multi-footprint LiDAR data in which the SNR was fixed for well-defined surfaces, and to carry out radiometric analyses with these data. Basic hypotheses concerning the influence of footprint diameter were verified, although comprehensive testing in tree canopies was left to the future. The datasets that have been acquired are unique as are the investigations performed using those data. Hopkinson (2007) studied the influence of footprint size and acquired dual-footprint data using a sensor in which the divergence could be altered. He reports that while all other parameters remained constant, the intensity values, instead of not changing in well-defined surfaces or changing by a factor of 0.38 in linear targets, reduced by a factor of 0.22 when the divergence was changed from 0.3 to $0.8 \mathrm{mrad}$.

The ALS60 sensor had a fixed beam divergence, but owing to the adjustable output power, footprint diameters ranging from 11 to $59 \mathrm{~cm}$ could be realized. The used power level was a compromise as over $50 \%$ more could have been used. The use of full power would have increased the signals by $3 \mathrm{~dB}$, but in that scenario, only $11 \mathrm{~dB}$ of power adjustment margin would have been available and the maximum acquisition height would have been less than $2 \mathrm{~km}$. Furthermore, it was recommended to reduce the power of the lowest acquisition height.

Unexpectedly the campaign planning software did not account for atmospheric losses and a $1 \mathrm{~dB}$ trend remained in the data. It caused the minimal echo-triggering target backscatter cross-section to increase with acquisition height and footprint size. A fairly realistic estimate of the atmospheric losses, $0.22 \mathrm{~dB} / \mathrm{km}$, was derived as a sub product of the radiometric normalization. Yet, it could not be ruled out that the observed trend was partially caused by systematic errors in the adjustment of the transmitted power.

Backscatter reflectance or peak amplitude normalization was done using natural surfaces assuming that the peak amplitude observations are in a linear relationship with the peak at-sensor power (irradiance). The linearity assumption had to be validated, using a separate multi-height LiDAR dataset, which was acquired for that purpose. The validation was based on the dependence between the power received and the spherical losses from well-defined surfaces. Fixed pulse power and receiver gain were applied and the signals from well-defined surfaces were, according to the radar equation, $1.6(2 \mathrm{~dB})$ times stronger from $700 \mathrm{~m}$ compared to $900 \mathrm{~m}$. Setting the output power and receiver gain correctly was crucial. While operating, the AGC circuit in the receiver can adjust and compensate the internal signal levels by up to $3 \mathrm{~dB}$. The brightest targets had pulses that were saturated and distorted in the $700 \mathrm{~m}$ DR intensity data. These targets were omitted from the calibration (Fig. 7), because it 
remained unclear if the saturation applied to the WFs as well. For example, dense canopies of (planophile) raspberry showed saturated intensity data. Because of the 2-dB difference in power received, the darkest objects that still triggered an echo in the $700 \mathrm{~m}$ data would not show in the $900 \mathrm{~m}$ data, and the brightest targets were rejected as the dynamic range of the receiver was exceeded in the $700 \mathrm{~m}$ acquisition. Natural and man-made surfaces were used in testing the linear response. The theoretical differences in power received are $23.4 \%$ and $26.5 \%$ between $700 \mathrm{~m}$ vs. $800 \mathrm{~m}$ and 800 vs. $900 \mathrm{~m}$, respectively. The withinsurface CVs of peak amplitude ranged from 3 to $15 \%$, which means that the estimation of the power term of the range normalization coefficient was susceptible to random errors. A 2\% error the mean peak amplitude (of a surface, e.g. in $800 \mathrm{~m}$ data) results in a coefficient estimate that is off by 0.2 . The influence of the incidence angle, or rather, the scan zenith angle, was weak and could be omitted in all analyses.

The resulting normalized peak amplitude data (Eq. 2) could be described as interval-scale observations of backscatter crosssection or reflectance in well-defined targets, at $1064 \mathrm{~nm}$. The smallest values corresponded to a reflectance of about 0.03 , which was observed in one very dark bitumen roof. This estimate was based on previous field measurements of HCRF carried out for research in the reflectance calibration of aerial images (Korpela et al., 2011). If full power had been applied (eye-safety, 3 dB higher), the echo-triggering reflectance would have been approximately $0.015-0.02$. In Korpela et al. (2013) it was found, using the 2012_10 $(22 \mathrm{~cm})$ data, that the minimum relative silhouette area in pine and spruce branches that produced an echo was about 10\%. Fig. 2 suggests that the ratio of mean peak amplitude in bitumen and pine crowns is about 2.5 and the $900 \mathrm{~nm}$ HCRF reference for the bitumen roof in Fig. 2 was 0.05 . If the reflectance of pine needles is $0.3-0.4$, the relative silhouette of 0.1 corresponds to a 'well-defined-surface reflectance' of about $0.03-0.04$. These calculations are coarse and inaccurate, but suggest that some coherence exists between earlier findings in canopies and those that were made here about well-defined surfaces.

The observations were shown to be influenced by instability of the transmitted power, in the low-power, low-altitude, smallfootprint data. The sensor manufacturer also pointed out this possibility and the repeated $500 \mathrm{~m}$ acquisitions in the following day also suggested this. It remained unclear what kind of temporal patterns these variations had, but at least a campaign-level offset was found. It may well be that the power fluctuations are smaller in sensors that apply a few fixed power levels and dualchannel receivers.

The impulse response and bandwidth characteristics were analyzed and only minor signal-strength dependent deformations of the WFs were observed in the 4 ns pulses. The deformations were neglected in the analyses here. Our experience with the Riegl LM S-Q680i sensor (cf. Armston et al., 2010) is worth noting here. The output power of LM S-Q680i has fixed levels and is tightly coupled with the pulse repetition frequency (PRF). Some low altitude (300 m) WF data were 'overexposed' and mapped to the non-linear part of the amplitude scale, which created an unwanted dependence of the echo width on signal strength, which was not present in a 700-m dataset acquired using the same PRF. LM S-Q680i has two receivers, and WF samples are stored from 
both receivers when necessary. Such dual-receiver, high- and low-gain designs are common and for example the new Leica ALS80-series sensors employ such a design. The gain difference of the channels is much larger than the $3 \mathrm{~dB}$ gain range of the AGC in ALS60. Compared with having two gain levels, the AGC in ALS60 could be considered a nuisance. In all, managing the wide dynamic range of LiDAR data acquisitions calls for different solutions, which are implemented in the transmitter and/or in the receiver. It would be better for radiometric analyses of LiDAR data if the sensor properties, i.e., an absolute calibration, were known. The reverse-engineering type of analyses that were carried out in this study would then not be needed for carrying out radiometrically quantitative LiDAR remote sensing.

The presented analyses revealed that the peak amplitude data in ALS60 was not in a very exact linear relationship with the power entering the aperture. However, this finding was obtained with the AGC-amplifier set at fixed gain and it cannot be ruled out that the AGC amplifier, when operating, causes further problems. However, this is unlikely, because the BW of such amplifiers can be expected to be larger than the BW of the digitizers or the photon detector. In Hovi et al. (2015), no evidence was found of systematic differences in peak amplitude or WF shape that were due to the two oscilloscopes behaving differently. As said, the oscilloscopes take turns in processing pulse pairs into WF data.

The dependence of 'unregistered' transmission losses on footprint size, preceding the triggering of waveform recording, was shown in power line cables and in pine crowns. The losses were from 2.6 to $0.5 \%$ in the cables and from 5 to $16 \%$ in the trees, for the footprint diameters of 11-59 cm. Canopies are comprised of gaps and clumped scatterers, while the cable is a single reflector obstructing the energy from reaching the ground, where the losses were detected as reductions in the average peak amplitude of hundreds of pulses. The sites used for loss detection had low and homogenous or at least spatially stationary ground reflectance variation. The influence of canopy transmission losses can be compensated to some degree in discretereturn (Korpela et al., 2012) and WF data (e.g. Richter et al., 2015) by imposing constraints such as constant reflectance or configuration of needles in a tree crown, which reduces the ambiguities related to backscatter cross-section. This was demonstrated by the comparison of the two bogs

The relative variation of peak amplitude was assessed in surfaces of varying small-scale structure (Section 2.8). Fine sand, which has a HCRF of about 0.2 at $1064 \mathrm{~nm}$, showed the lowest CV, $4.5 \%$ in $11-\mathrm{cm}$ data, and as low as $2.8 \%$ in the $59-\mathrm{cm}$ data. The target was a volleyball field with a 'moderately bumpy' surface and a few dry leafs blended in the sand. Bitumen was least influenced by footprint size, but the CVs were higher, on the order of $9 \%$, because of the low reflectance. The results were logical, concerning the influence of the footprint size and the averaging effect in large footprint data. The observed CVs suggest that the instrument is rather stable or precise as there were always data from two or more strips per test surface and footprint size.

Regarding the influence of the footprint size on the tested first-echo WF attributes in tree canopies, there were minor speciesspecific differences or 'signatures'. For example, the peak amplitude decreased most in Scots pine stand, the structure of which 
is less clumped or 'more diffuse' compared to Norway spruce. The reduction in peak amplitude with increasing footprint size was smallest in the case of birch, which have compact crowns at the age of 30 years. Actually these findings that relate to canopy structure are line with those concerning the response of intensity data to R variation (Korpela et al., 2010a; Gatsiolis, 2011), because the decrease of power received that is due to an increase of divergence is also dependent on the structure (Section 1). As hypothesized, the echo width increased with footprint size and the relative increase was largest in the canopy of spruce, which is reasonable, given the 'clumped structure' and 'layered compact branches'. The energy was the largest in birch, and this could possibly be due to the slightly higher reflectance of the leaves. Then again, backscattering in birch is strongly influenced by leaf arrangement as well (Hovi and Korpela, 2014). The tests in forest canopies were hampered by the remaining between-dataset SNR differences, which were subtracted using the roads adjacent to the test stands as control surfaces.

This study showed that it is possible to acquire multi-footprint data that is radiometrically comparable for well-defined surfaces, using a commercial topographic LiDAR sensor. The study has shown how to carry out careful analyses to verify that the WF amplitude observations are on the interval scale. The impulse response and bandwidth characteristics of the sensor were tested in a simple manner to verify that the WF features depend only on the target-pulse interaction, in order to draw correct

\section{Acknowledgements}

Advice about the ALS60 sensor by Dr. Ron Roth at Leica Geosystems was indispensable. I also thank the personnel at Finnmap in Helsinki, especially M r. Jonne Davidsson, Mr. Felix Rohrbach and M r. Jussi Havia, who arranged the unique data acquisitions with the ALS60. Dr. Aarne Hovi wrote the original WF attribute extraction code. Dr. Kyle Eyvindson, Dr. Reija Haapanen and Dr. Kim Östman kindly revised the text. The Academy of Finland provided the funding for this work. 
Armston, J., Disney, M., Lewis, P., Scarth, P., Phinn, S., Lucas, R., Bunting, P. \& Goodwin, N. (2013). Direct retrieval of canopy gap probability using airborne waveform lidar. Remote Sensing of Environment 134:24-38.

Disney, M.I., Kalogirou, V., Lewis, P., Prieto-Blanco, A., Hancock, S. \& Pfeifer, M. (2010) Simulating the impact of discrete-return lidar system and survey characteristics over young conifer and broadleaf forests, Remote Sensing of Environment 114(7): $1546-1560$.

Gatziolis, D. (2011). Dynamic range-based intensity normalization for airborne, discrete return LiDAR data for forest canopies. Photogrammetric Engineering \& Remote Sensing. 77(3): 251-259.

Goodwin, N.R., Coops, N.C. \& Culvenor, D.C. (2006). Assessment of forest structure with airborne LiDAR and the effects of

Hovi, A. \& Korpela, I. (2014). Real and simulated waveform-recording LiDAR data in juvenile boreal forest vegetation. Remote

Hopkinson, C. (2007). The influence of flying altitude, beam divergence, and pulse repetition frequency on laser pulse return intensity and canopy frequency distribution. Canadian Journal of Remote Sensing 33: 312-324.

Sensing of Environment 140: 665-678.

Hovi A., Korhonen L., Vauhkonen J. \& Korpela I. (2015). LiDAR waveform features for tree species classification and their sensitivity to tree- and acquisition related parameters. Remote Sensing of Environment 173: 224-237.

Hovi, A. (2015). Towards an enhanced understanding of airborne LiDAR measurements of forest vegetation. Dissertationes Forestales 200. $69 \mathrm{p}$.

Korpela, I. (2008). Mapping of understory lichens with airborne discrete-return LiDAR data. Remote Sensing of Environment 112(10): 3891-3897.

Korpela, I., Ørka H.O., Heikkinen V., Tokola T., \& Hyyppä J. (2010a). Range- and AGC normalization of LIDAR intensity data for vegetation classification. ISPRS Journal of Photogrammetry and Remote Sensing 65(4): 369-379.

Korpela, I., Ørka, H.O., M altamo, M., Tokola, T. \& Hyyppä, J. (2010b). Tree species classification using airborne LiDAR - effects of stand and tree parameters, downsizing of training set, intensity normalization and sensor type. Silva Fennica 44(2): 319-339

Korpela, I., Heikkinen, V., Honkavaara, E., Rohrbach F. \& Tokola, T. (2011). Variation and anisotropy of reflectance of forest trees in radiometrically calibrated airborne line sensor images - implications for species classification in digital aerial images. Remote Sensing of Environment 115(8): 2062-2074. 
561 Korpela I., Hovi A. \& M orsdorf F. (2012). Understory trees in airborne LiDAR data - Selective mapping due to transmission losses 562 and echo-triggering mechanisms. Remote Sensing of Environment 119: 92-104.

563 Korpela I., Hovi A. \& Korhonen L. (2013). Backscattering of individual LiDAR pulses explained by photogrammetrically derived 564 vegetation structure. ISPRS journal of photogrammetry and remote Sensing 83: 81-93.

565 Maltamo M. \& Packalén P. (2014). Species-specific management inventory in Finland. In: Maltamo M., Næsset E., Vauhkonen J. 566 (eds.). Forestry applications of airborne laser scanning: Concepts and case studies. Springer, The Netherlands, p. 241-252

567 Mallet, C. \& Bretar, F. (2009). Full-waveform topographic lidar: State-of-the-art, ISPRS journal of photogrammetry and remote 568 sensing 64(1):1-16.

569 Ørka, H-O., Næsset, E., Bollandsås, O., M. (2010). Effects of different sensors and leaf-on and leaf-off canopy conditions on echo 570 distributions and individual tree properties derived from airborne laser scanning. Remote sensing of environment 114(7): 1445$571 \quad 1461$.

572 Richter, K., Blaskow, R., Stelling, N., \& M aas, H. G. (2015). Reference Value Provision Schemes for Attenuation Correction of Full573 Waveform Airborne Laser Scanner Data. ISPRS Ann. Photogramm. Remote Sens. Spat. Inf. Sci. 1: 65-72.

574 Roncat, A., Briese, C., Jansa, J. \& Pfeifer, N. (2014). Radiometrically calibrated features of full-waveform lidar point clouds based 575 on statistical moments. IEEE Geoscience and remote sensing letters, 11(2): 549-553

576 Wagner W., Ullrich A., Ducic V., Melzer T., Studnicka N. (2006). Gaussian decomposition and calibration of a novel small577 footprint full-waveform digitising airborne laser scanner. ISPRS Journal of Photogrammetry and Remote Sensing 60: 100-112.

578 Wagner, W. (2010). Radiometric calibration of small-footprint full-waveform airborne laser scanner measurements: Basic 579 physical concepts, ISPRS Journal of Photogrammetry and Remote Sensing 65 (6): 505-513.

580 Vauhkonen, J., M altamo, M., M CRoberts, R.E., \& Naesset, E. (2014). Introduction to forestry applications of airborne laser 581 scanning. In: M altamo M., Næsset E., Vauhkonen J. (eds.). Forestry applications of airborne laser scanning: Concepts and case 582 studies. Springer, The Netherlands, p. 1-16. 